\title{
Broad Bandwidth Optical and Mechanical Rheometry of Wormlike Micelle Solutions
}

\author{
N. Willenbacher, ${ }^{*}$ C. Oelschlaeger, and M. Schopferer \\ Institute of Mechanical Process Engineering and Mechanics, Universität Karlsruhe, 76131 Karlsruhe, Germany \\ P. Fischer \\ Institute of Food Science and Nutrition, ETH Zurich, 8092 Zurich, Switzerland \\ F. Cardinaux and F. Scheffold ${ }^{\dagger}$ \\ Department of Physics, University of Fribourg, 1700 Fribourg Perolles, Switzerland
}

\begin{abstract}
We characterize the linear viscoelastic shear properties of an aqueous wormlike micellar solution using diffusing wave spectroscopy (DWS) based tracer microrheology as well as various mechanical techniques such as rotational rheometry, oscillatory squeeze flow, and torsional resonance oscillation covering the frequency range from $10^{-1}$ to $10^{6} \mathrm{rad} / \mathrm{s}$. Since DWS as well as mechanical oscillatory squeeze flow and torsional resonance oscillation cover a sufficiently high frequency range, the persistence length of wormlike micelles could be determined directly from rheological measurements for the first time.
\end{abstract}

PACS numbers: 83.80.Qr, 82.70.-y, 83.60.Bc, 83.85.Ei

Significant progress has been made over the past decade in developing optical microrheology as a noninvasive means to study the rheological properties of soft complex fluids. Following the seminal Letter of Mason and Weitz in 1995 [1], several hundred studies have reported on the application of optical microrheology to such diverse systems as polymers, emulsions, gels, biomaterials, hydrogel scaffolds, stomach mucus, magnetic fluids, ceramics, slurries, and many more [1-5]. The underlying idea is to study the response of small (colloidal) particles embedded in the system under study. The motion of probe particles can either be controlled actively, e.g., using optical tweezers or one can analyze the thermal motion of particles to obtain information about the viscoelastic properties of the surrounding fluid. The latter can be achieved by using diffusing wave spectroscopy (DWS) [6]. DWS provides a fast ensemble average of the tracer motion and can resolve extremely fast displacements on the order of microseconds with subnanometer resolution. Despite the large literature, benchmarking optical microrheological techniques with macroscopic mechanical measurements is still not concluded. Almost all comparisons between microrheology and macrorheology have been restricted to the frequency range $<10^{2} \mathrm{rad} / \mathrm{s}$ due to mechanical limitations and inertial effects in the gap loading limit of conventional rotational rheometers and the limited availability of mechanical devices operating at higher frequencies [7-9].

Here we apply both DWS and macroscopic mechanical rheometry to characterize an aqueous solution of cetylpyridinium chloride and sodium salicylate $(100 \mathrm{~m} M$ CPyCl-60m $M$ NaSal) at different temperatures. These solutions display complex viscoelastic properties but are easy and reproducible to prepare and stable in time. They exhibit fast dynamics and the structural features are much smaller than the tracer particles. The latter is an important requirement for the successful application of tracer microrheology which might otherwise lead to erroneous results [4]. Because of the peculiar viscoelastic behavior such systems have found wide commercial application ranging from personal care to enhanced oil recovery products [10]. Rapid access to microscopic structural and dynamic properties is therefore of interest both from a fundamental and an applied point of view. DWS is especially suited to yield fast and accurate results at high frequencies up to $10^{6} \mathrm{rad} / \mathrm{s}$. In order to access intermediate and high frequencies by mechanical rheometry we use a piezodriven squeeze-flow device in combination with two torsional resonance oscillators to extend the available frequency range up to $3 \times 10^{5} \mathrm{rad} / \mathrm{s}$ [11].

At the concentrations and temperatures investigated here, the surfactant molecules form semiflexible, cylindrical aggregates, so-called wormlike micelles, exhibiting unique structural and rheological properties [12-14]. Characteristic structural parameters are the diameter of the micelles $d_{\text {mic }}$, their contour length $L$, which is related to the scission energy $E_{c}$ necessary to create two micellar end caps and the persistence length $l_{p}$, which is related to the bending modulus $\kappa=k_{B} T l_{p}$, with the Boltzmann constant $k_{B}$ and the temperature $T$. $l_{p}$ strongly depends on the nature of the surfactant and the counterion, their concentration and mixing ratio, as well as the ionic strength of the solution. Mostly $l_{p}$ has been deduced from small-angle neutron scattering, but it can also be extracted from the dynamic structure factor accessible by neutron spin echo measurements [15]; appropriate models have to be applied, and uncertainties occur if data have to be extrapolated beyond the experimentally accessible $q$ range. Using birefringence measurements, knowledge about stress-optical 
coefficients is needed, which is not always accessible [16]. Dynamic light scattering provides the appropriate $q$ range only if the micelles are sufficiently stiff [17].

For entangled solutions the contour length between two entanglements $l_{e}$ or the mesh size $\xi$ is another important structural feature. Stress relaxation then occurs via reptation and scission [12]. The structural parameters $L, l_{e}$, and $\xi$ are related to the low and intermediate frequency dynamics of the system. In the high frequency regime stress relaxes via intramicellar processes: First dominated by the Rouse-Zimm modes and then by internal relaxation modes of individual Kuhn segments. In this frequency range $G^{*}=$ $G^{\prime}+i G^{\prime \prime}$ exhibits a power-law behavior $G^{*} \sim \omega^{a}$ with the exponent $a$ changing from approximately $5 / 9$ in the Rouse-Zimm regime to $3 / 4$ where internal bending modes of Kuhn segments dominate [18]. The change in the exponent occurs around a critical frequency $\omega_{0}=k T / 8 \eta_{s} l_{p}^{3}$ corresponding to the shortest relaxation time in the RouseZimm spectrum, and $l_{p}$ can be deduced directly from rheological experiments either by determination of $\omega_{0}$ or from the absolute values of $G^{*}$ in the frequency range $\omega \gg$ $\omega_{0}$. These scaling concepts have been proven experimentally in various studies on biopolymer solutions [19-21]. These systems exhibit typical $l_{p}$ values of several micrometers and, accordingly, the $\omega^{3 / 4}$ scaling for $G^{*}$ sets in at frequencies between 10 and $10^{3} \mathrm{rad} / \mathrm{s}$. Typical values for the persistence length of wormlike micelles are expected below $100 \mathrm{~nm}$; correspondingly, the $\omega^{3 / 4}$ scaling regime should occur in the frequency range beyond $10^{4} \mathrm{rad} / \mathrm{s}$. In this Letter we demonstrate that accurate high frequency rheology can be used as a powerful laboratory tool to determinate characteristic structural and dynamic properties of surfactant solutions. With our combined mechanical and optical approach we can accurately determine $E_{c}$, and, moreover, the persistence length $l_{p}$ is accessible for solutions of wormlike surfactant micelles or other semiflexible objects down to $l_{p}$ values of about $1 \mathrm{~nm}$.

Echo two-cell DWS experiments in transmission geometry were performed as described in [5]. Typical measurement times were in the range of 3-5 min. Standard glass cuvettes (Hellma) with path lengths 2 or $5 \mathrm{~mm}$ and a width of $10 \mathrm{~mm}$ were used. $1 \%-2 \%$ of polystyrene sulfonate particles (diameter $720 \mathrm{~nm}$, IDC Corporation, Portland, U.S.A.) were used as tracers. From the DWS intensity correlation function we obtain the ensemble averaged particle mean square displacement $\left\langle\Delta r^{2}(t)\right\rangle$ [6]. From a fit to $\ln \left\langle\Delta r^{2}(\ln t)\right\rangle$ with a polynomial of order 6 or 7 we calculate the viscoelastic moduli $G^{\prime}(\omega)$ and $G^{\prime \prime}(\omega)$ from the generalized Stokes-Einstein relation following the approach of Mason et al. [21]. We have checked that the choice of the polynomial order and the range of data selected do not influence the results. At times shorter than $10^{-5} \mathrm{~s}$, or frequencies above $\omega=10^{5} \mathrm{rad} / \mathrm{s}$, inertia effects become sizable [22]. To get access to this interesting ultrahigh frequency regime we introduce a selfconsistent correction scheme for $\left\langle\Delta r^{2}(t)\right\rangle$. The high fre- quency viscosity $\eta_{\text {eff }}$ determines the influence of fluid inertia on particle displacement, and as long as the high frequency behavior is predominately viscous $G^{\prime \prime}(\omega) \gg$ $G^{\prime}(\omega)$ we can correct the experimental data using the analytical expression for Newtonian fluids derived by Hinch [22]. In practice we correct the experimental particle mean square displacement: $\left\langle\Delta r^{2}(t)\right\rangle=f(t)\left\langle\Delta r^{2}(t)\right\rangle_{\text {DWS }}$ with $f(t)$ from [22] and take an initial guess value of $\eta_{\text {eff }} \approx$ $3 \mathrm{mPa}$ s from mechanical rheometry data at the highest frequency accessible. Thus we obtain a corrected $G^{*}(\omega)$ and use the corresponding $G^{\prime \prime}=\eta \omega$ to recalculate $f(t)$. This procedure is repeated two or three times until the result converges [23].

A rotational rheometer, Rheometric Scientific ARES, equipped with a cone-plate sample cell was used for oscillatory shear experiments covering the frequency range from 0.01 to $100 \mathrm{rad} / \mathrm{s}$. Oscillatory squeeze-flow experiments were performed in the frequency range from 0.2 to $10^{4} \mathrm{rad} / \mathrm{s}$ using a piezodriven axial vibrator. Torsional resonance oscillation was performed at four distinct frequencies $\left[(50,119,195\right.$, and 364$\left.) \times 10^{3} \mathrm{rad} / \mathrm{s}\right]$. The principle of measurement, the mechanical and electronic setup, as well as the calibration procedure for both techniques have been described in detail elsewhere [11]. Strain amplitudes in all mechanical experiments were always low enough to ensure linear sample response.

Dynamic shear moduli $G^{\prime}$ and $G^{\prime \prime}$ at $T=20^{\circ} \mathrm{C}$ obtained from mechanical rheometry and DWS are compared in Fig. 1. The shapes of the relaxation spectra from DWS coincide very well with those from mechanical rheometry, but the absolute values of the $G^{\prime}$ and $G^{\prime \prime}$ data are about $10 \%-20 \%$ lower than those from mechanical measurements. Taking into account the uncertainties in experiment as well as the approximations included in the data analysis we consider this as good agreement. Nevertheless, deviations in the $G^{\prime \prime}$ data occur especially around the first crossover region where $G^{\prime \prime}$ has a maximum and around the minimum in $G^{\prime \prime}$ at intermediate frequencies. The latter may be rationalized by the following arguments: In an oscillatory shear experiment the real and imaginary parts of $G^{*}(\omega)$ are determined from the amplitude and phase shift of the response signal. Such a phase analysis is much more accurate than the DWS data treatment, where the $\left\langle\Delta r^{2}(t)\right\rangle$ is measured in time space. Small deviations around the first crossing point of $G^{\prime}$ and $G^{\prime \prime}$ are probably residual effects of the polynomial fitting.

Irrespective of these deviations, various important features of the relaxation spectra of wormlike micellar solutions can be equally well deduced from both methods. The characteristic first and second crossover frequencies $\left[G^{\prime}\left(\omega_{i}\right)=\left.G^{\prime \prime}\left(\omega_{i}\right)\right|_{i=r, e}\right]$ are summarized in Table I together with the plateau modulus $G_{0}$ and the ratio of $G_{0} / G_{\min }^{\prime \prime} . G_{0}$ is determined as the value of the modulus $G^{\prime}$ at the frequency at which $G^{\prime \prime}$ has its local minimum $G_{\min }^{\prime \prime}$. The ratio $G_{0} / G_{\min }^{\prime \prime}$ is another important rheological quantity for wormlike micelles, since it is directly related 


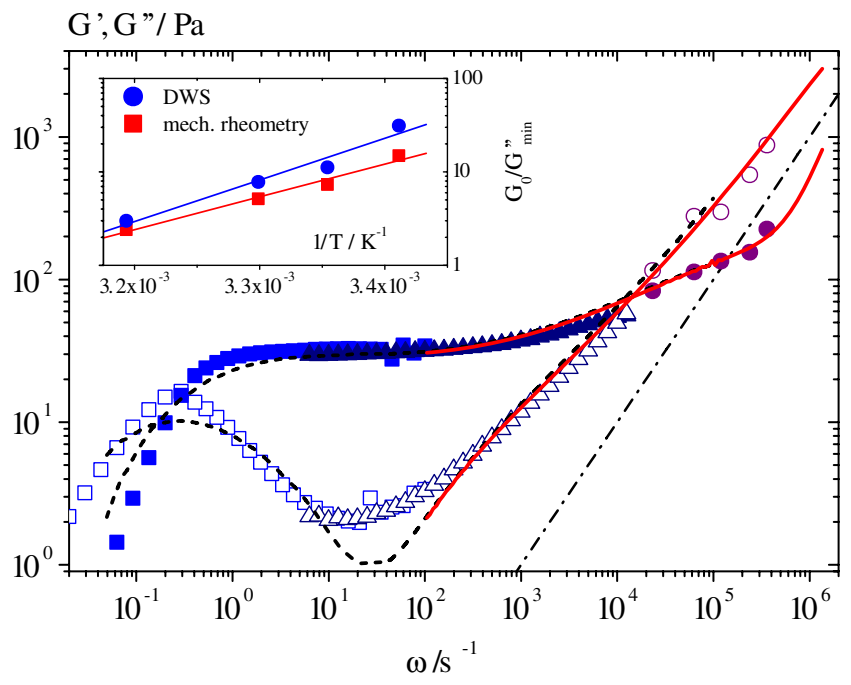

FIG. 1 (color online). Dynamic shear moduli $G^{\prime}$ and $G^{\prime \prime}$ of an aqueous solution of $100 \mathrm{~m} M \mathrm{CPyCl}$ and $60 \mathrm{~m} M \mathrm{NaSal}$ at $T=$ $20^{\circ} \mathrm{C}$ obtained from DWS (dashed lines and solid lines from two different measurements) and various mechanical rheometers $\left(G^{\prime}\right.$, solid symbols; $G^{\prime \prime}$, open symbols). Rotational rheometer (squares), oscillatory squeeze flow (triangles), torsional resonators (circles). The modulus $G^{\prime \prime}=\omega \eta_{s}$ of water is included for reference (dash-dotted line). Inset: Ratio of the plateau modulus $G_{0}$ and the minimum value of $G^{\prime \prime}$ as a function of $1 / T$ for the same solution.

to the ratio $L / l_{e} \sim \exp \left(E_{c} / 2 k_{B} T\right)$, and its $T$ dependence is used to determine $E_{c}$ [12]. The values from both methods are in excellent agreement.

The terminal zone and accordingly $\omega_{r}$ are strongly shifted to higher frequencies and $G_{\min }^{\prime \prime}$ strongly increases as temperature is raised, directly showing that the micellar contour length $L \sim \omega_{r}^{-3}$ decreases as temperature goes up. $G_{0}$ and hence the mesh size $\xi$ of the entanglement network are essentially independent of $T$. Accordingly, the zero shear viscosity $\eta_{0}=G_{0} / \omega_{r}$ strongly decreases with increasing temperature. Similar behavior has been reported for other surfactant solutions [14]. The high frequency dynamic shear moduli are essentially independent of temperature. A closer inspection of the data reveals a scaling $G^{\prime \prime} \sim \omega^{3 / 4}$ for $\omega>10^{4} \mathrm{rad} / \mathrm{s}$ and indicates the onset of a $G^{\prime} \sim \omega^{3 / 4}$ scaling for $\omega>5 \times 10^{5} \mathrm{rad} / \mathrm{s}$ [7]. This is consistent with the predictions for wormlike chains [18]. The frequency range where internal bending modes of individual Kuhn segments dominate is reached here and covers about two decades in frequency.
The plateau modulus $G_{0}$ from mechanical rheometry is in excellent agreement with [13] and yields a mesh size of $\xi=52 \pm 2 \mathrm{~nm}$. Since $\xi$ and $l_{p}$ are almost independent of temperature we can assume this also for $l_{e}$. Consequently, the temperature dependence of $G_{0} / G_{\min }^{\prime \prime}=L / l_{e}$ is determined by the change in $L$, and the end cap energy $E_{c}$ can be extracted from the slope of a semilogarithmic plot of $G_{0} / G_{\min }^{\prime \prime}$ versus $1 / T$ [12]. From mechanical rheometry data (see Table I and Fig. 1) we obtain $E_{c}=(23 \pm$ 2.4) $k T$, similar values have been reported for other surfactants [14].

The persistence length $l_{p}$ is determined from the $G^{\prime \prime}-$ $\omega \eta_{s}$, where $\eta_{s}$ is the solvent viscosity. We fit the function $G^{\prime \prime}-\omega \eta_{s}=k_{\mathrm{Dws}} \omega^{3 / 4}$ to the experimental data as shown in Fig. 2 and analyze the result in two different ways. Gittes and MacKintosh derived the following relation based on a statistical mechanical treatment of the single filament stress response of semiflexible chains [18]:

$$
G^{*}-i \omega \eta_{s}=\frac{1}{15} \rho \kappa l_{p}\left(\frac{-2 i \zeta}{\kappa}\right)^{3 / 4} \omega^{3 / 4},
$$

where $\zeta$ is the lateral drag coefficient and $\rho$ is the area density of micelles. For $\mathrm{CPyCl} / \mathrm{NaSal}$ we take $d_{\text {mic }}=$ $2.5 \mathrm{~nm}$ [13] to calculate $\rho$, and according to [18] we insert $\zeta=4 \pi \eta_{s} / \ln \left(0.6 \lambda / d_{\text {mic }}\right) \approx 0.005 \mathrm{Ns} / \mathrm{m}^{2}$ for the lateral drag coefficient. For the characteristic length scale $\lambda$ we choose here the mesh size $\xi$. Comparing then the prefactor in Eq. (1) to the value for the fit parameter $k_{\mathrm{DwS}}$ results in $l_{p}=32 \pm 2 \mathrm{~nm}$. As a consistency check we can take this value to estimate the frequency $\omega_{0}$ at which the data should start to deviate from the $3 / 4$ scaling [Eq. (1)]. We find a deviation of $10 \%$ for the data set shown in Fig. 2. Alternatively, we can extract $\omega_{0}$ from the crossover frequency of the $\omega^{5 / 9}$ and $\omega^{3 / 4}$ scaling regime observed in the mechanical data, as shown in the inset of Fig. 2. We obtain $\omega_{0}=(22 \pm 3) \times 10^{3} \mathrm{rad} / \mathrm{s}$ and $l_{p}=29 \pm 3 \mathrm{~nm}$ at $20^{\circ} \mathrm{C}$.

In conclusion, we show that DWS microrheology can provide results in quantitative agreement with bulk mechanical measurements of $G^{\prime}$ and $G^{\prime \prime}$ in the frequency range from $10^{-1}$ to $10^{6} \mathrm{rad} / \mathrm{s}$. As a consequence, DWS as well as mechanical high frequency rheometry can be used as a powerful tool to determine characteristic features of wormlike micelles, like $E_{c}$ and $\xi$ as well as $l_{p}$ directly from rheological measurements. With both approaches $l_{p}$ is accessible for aqueous solutions of wormlike surfactant micelles or other semiflexible objects down to $l_{p} \approx 1 \mathrm{~nm}$

TABLE I. Crossover frequencies $\omega_{r}$ and $\omega_{e}$ as well as plateau moduli $G_{0}$ and $G_{0} / G_{\min }^{\prime \prime}$ from mechanical rheometry and DWS.

\begin{tabular}{lcccccccc}
\hline \hline \multicolumn{1}{c}{$\left.{ }^{\circ} \mathrm{C}\right)$} & $\omega_{r}\left(\mathrm{~s}^{-1}\right)$ & $\omega_{e}\left(\mathrm{~s}^{-1}\right)$ & $G_{0}(\mathrm{~Pa})$ & $G_{0} / G_{\min }^{\prime \prime}$ & $l_{p}(\mathrm{~nm})$ & $\omega_{r}\left(\mathrm{~s}^{-1}\right)$ & \multicolumn{2}{c}{ DWS microrheology } \\
\hline 20 & 0.3 & 11860 & 31 & 14.9 & $29 \pm 3$ & 0.2 & 11870 & 31.3 \\
25 & 0.6 & 11100 & 32 & 7.4 & $27 \pm 3$ & 0.5 & 12737 & 11.2 \\
30 & 1.9 & 11200 & 28 & 5.2 & $26 \pm 3$ & 1.2 & 14120 & $31 \pm 2$ \\
40 & 16 & 11946 & 27 & 2.4 & - & 12 & 15560 & $30 \pm 3$ \\
\hline \hline
\end{tabular}




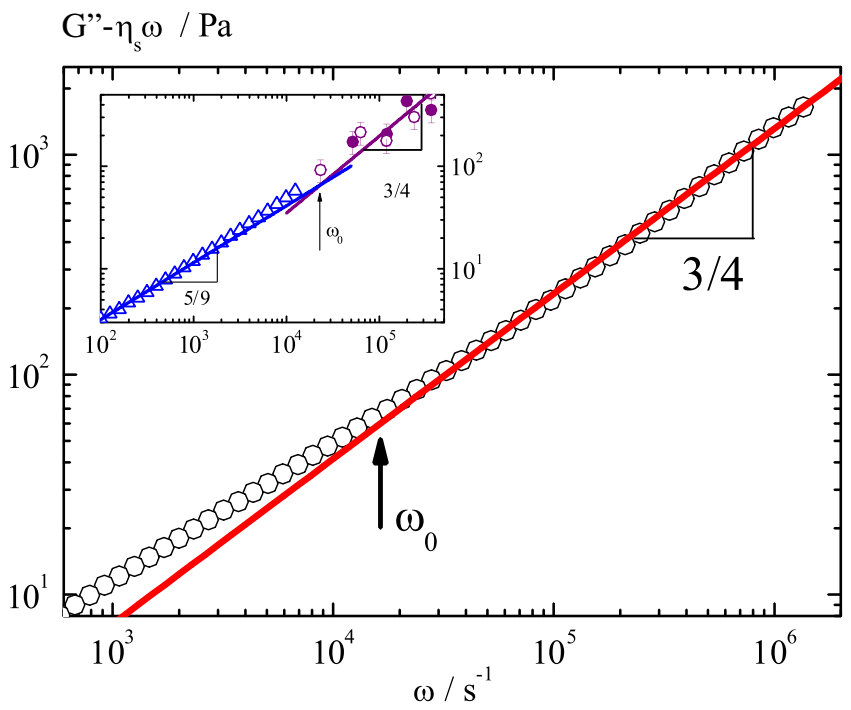

FIG. 2 (color online). Loss modulus $G^{\prime \prime}-\omega \eta_{s}$ of an aqueous solution of $100 \mathrm{~m} M \mathrm{CPyCl}$ and $60 \mathrm{~m} M \mathrm{NaSal}$ at $T=20^{\circ} \mathrm{C}$ as obtained from DWS. Solid line: fit of $G^{\prime \prime}=k_{\mathrm{DWS}} \omega^{3 / 4}$ to the data for $\omega>4 \times 10^{4} \mathrm{rad} / \mathrm{s}$. Inset: Oscillatory squeeze-flow (triangles) and torsional resonance oscillation (circles) data. Open circles mark data obtained using the resonators described in [11]. Solid lines: fit of different power laws to experimental data.

and may, e.g., also be applied to test scaling theories for the electrostatic contribution to the persistence length of ordinary polyelectrolytes.

Discussions with G. Koenderink, F. MacKintosh, R. Prud'homme, C. Schmidt, E. Frey, and P. Schurtenberger, as well as financial support by the Swiss National Science Foundation, are gratefully acknowledged.

*norbert.willenbacher@ciw.uni-karlsruhe.de ${ }^{\dagger}$ Frank.Scheffold@unifr.ch

[1] T. G. Mason and D. A. Weitz, Phys. Rev. Lett. 74, 1250 (1995); T. G. Mason, H. Gang, and D. A. Weitz, J. Opt. Soc. Am. A 14, 139 (1997).

[2] B. Schnurr, F. Gittes, F. C. MacKintosh, and C. F. Schmidt, Macromolecules 30, 7781 (1997).

[3] F. Cardinaux et al., Europhys. Lett. 57, 738 (2002).

[4] J. C. Crocker et al., Phys. Rev. Lett. 85, 888 (2000); D. T. Chen et al., Phys. Rev. Lett. 90, 108301 (2003).

[5] P. Zakharov, F. Cardinaux, and F. Scheffold, Phys. Rev. E 73, 011413 (2006).

[6] G. Maret and P. E. Wolf, Z. Phys. B 65, 409 (1987); D. J. Pine, D. A. Weitz, P. M. Chaikin, and E. Herbolzheimer, Phys. Rev. Lett. 60, 1134 (1988).

[7] P. Hebraud et al. were the first to compare conventional and DWS based rheometry at elevated frequencies up to $\omega=6 \times 10^{4} \mathrm{rad} / \mathrm{s}$ [8]. The droplet size of the emulsions studied by light scattering and those studied by piezodriven squeeze-flow rheometry were, however, different. Further, the interpretation of the DWS data is more difficult since the droplets under study cannot be considered independent tracers. Another recent comparative study on wormlike micelle solution has been reported by Buchanan et al. [9]. Their experiments were limited to frequencies below $\omega=10^{5} \mathrm{rad} / \mathrm{s}$. They could not resolve the $\omega^{3 / 4}$ scaling and hence could not deduce $l_{p}$. In contrast to our system their samples were prepared in $0.5 \mathrm{M} \mathrm{NaCl}$ solution. Such a high ionic strength reduces the stiffness of the micelles, and the $\omega^{3 / 4}$ scaling is expected to occur at frequencies beyond $10^{5} \mathrm{rad} / \mathrm{s}$.

[8] P. Hebraud, F. Lequeux, and J. F. Palierne, Langmuir 16, 8296 (2000).

[9] M. Buchanan, M. Atakhorrami, J.F. Palierne, F. C. MacKintosh, and C.F. Schmidt, Phys. Rev. E 72, 011504 (2005).

[10] L. M. Walker, Curr. Opin. Colloid Interface Sci. 6, 451 (2001).

[11] G. Fritz, W. Pechold, N. Willenbacher, and N. J. Wagner, J. Rheol. (N.Y.) 47, 303 (2003); J. J. Crassous, R. Regisser, and M. Ballauff, J. Rheol. (N.Y.) 49, 851 (2005).

[12] M.E. Cates and S. J. Candau, J. Phys. Condens. Matter 2, 6869 (1990); R. Granek and M. E. Cates, J. Chem. Phys. 96, 4758 (1992).

[13] H. Rehage and H. Hoffmann, Mol. Phys. 74, 933 (1991).

[14] S. J. Candau et al., Langmuir 5, 1225 (1989); A. Khatory et al., Langmuir 9, 1456 (1993); I. Couillet et al., Langmuir 20, 9541 (2004).

[15] L. J. Magid, Z. Li, and P. D. Butler, Langmuir 16, 10028 (2000); L. Arleth, M. Bergström, and J.S. Pedersen, Langmuir 18, 5343 (2002); C. Sommer et al., Langmuir 18, 2495 (2002); Seto et al., J. Phys. Chem. Solids 60, 1371 (1999).

[16] E. K. Wheeler, P. Izu, and G. G. Fuller, Rheol. Acta 35, 139 (1996); T. Shikata, S. J. Dahman, and D. S. Pearson, Langmuir 10, 3470 (1994).

[17] H. von Berlepsch, L. Harnau, and P. Reineker, J. Phys. Chem. B 102, 7518 (1998).

[18] F. Gittes and F. C. MacKintosh, Phys. Rev. E 58, R1241 (1998); D. Morse, Macromolecules 31, 7030 (1998).

[19] R. W. Rosser et al., Macromolecules 11, 1239 (1978).

[20] G. H. Koenderink, M. Atakhorrami, F. C. MacKintosh, and C. F. Schmidt, Phys. Rev. Lett. 96, 138307 (2006).

[21] T. G. Mason, K. Ganesan, J. H. van Zanten, D. Wirtz, and S. C. Kuo, Phys. Rev. Lett. 79, 3282 (1997).

[22] D. A. Weitz, D. J. Pine, P. N. Pusey, and R. J. A. Tough, Phys. Rev. Lett. 63, 1747 (1989); A. J. C. Ladd, H. Gang, J. X. Zhu, and D. A. Weitz, Phys. Rev. Lett. 74, 318 (1995); E. Hinch, J. Fluid Mech. 72, 499 (1975).

[23] It is worthwhile to comment on the general validity of this procedure. At frequencies above $10^{4} \mathrm{rad} / \mathrm{s}$ the viscous component $G^{\prime \prime}$ is usually higher than the elastic $G^{\prime}$ for most soft materials. Allowing for a frequency dependence of the viscosity is an ad hoc extension of the Hinch formula we expect to work well since the actual frequency dependence is generally weak in the high frequency range, and as long as $\omega<5 \times 10^{7} \mathrm{rad} / \mathrm{s} f(t)$ never exceeds a factor of 2 for submicron sized particles even for low viscosity fluids. Higher viscosity reduces the correction and in our case we always find $f(t)<1.4$. 